\title{
New metabolic tracers for detectable PSA levels in the post- prostatectomy setting: is the era of melting glaciers upcoming?
}

\author{
Rosario Mazzola ${ }^{1}$, Francesco Cuccia ${ }^{1}$, Vanessa Figlia ${ }^{1}$, Niccolò Giaj-Levra ${ }^{1}$, Luca Nicosia ${ }^{1}$, \\ Francesco Ricchetti ${ }^{1}$, Michele Rigo ${ }^{1}$, Nadia Pasinetti ${ }^{2}$, Matteo Salgarello ${ }^{3}$, Filippo Alongi ${ }^{4,5}$ \\ ${ }^{1}$ Advanced Radiation Oncology Department, IRCCS Sacro Cuore Don Calabria Hospital, Negrar-Verona, Italy; ${ }^{2}$ Department of Radiation \\ Oncology, Ospedale di Esine - ASL Vallecamonica-Sebino, Esine, Italy; ${ }^{3}$ Nuclear Medicine, IRCCS Sacro Cuore Don Calabria Hospital, Negrar- \\ Verona, Italy; ${ }^{4}$ Advanced Radiation Oncology Department, IRCCS Sacro Cuore Don Calabria Hospital, Negrar-Verona, Italy; ${ }^{5}$ University of Brescia, \\ Brescia, Italy \\ Correspondence to: Dr. Nadia Pasinetti. Department of Radiation Oncology, Ospedale di Esine - ASL Vallecamonica-Sebino, Esine, Italy. \\ Email: nadia.pasinetti@unibs.it. \\ Provenance: This is an invited article commissioned by the Section Editor Dr. Xiao Li (Department of Urology, Jiangsu Cancer Hospital \& Jiangsu \\ Institute of Cancer Research \& Affiliated Cancer Hospital of Nanjing Medical University, Nanjing, China). \\ Comment on: Calais J, Ceci F, Eiber M, et al. ${ }^{18} \mathrm{~F}$-fluciclovine PET-CT and ${ }^{68} \mathrm{Ga}$-PSMA-11 PET-CT in patients with early biochemical recurrence \\ after prostatectomy: a prospective, single-centre, single-arm, comparative imaging trial. Lancet Oncol 2019;20:1286-94.
}

Submitted Aug 18, 2019. Accepted for publication Dec 05, 2019.

doi: $10.21037 /$ tau.2019.12.34

View this article at: http://dx.doi.org/10.21037/tau.2019.12.34

The introduction of new metabolic tracers for positron emission tomography (PET) imaging is changing the landscape in the early biochemical recurrence of prostate cancer after radical prostatectomy (1).

Current management of the recurrent disease is determined by location and extent (2); consequently, the role of adequate imaging becomes crucial for the diagnostic assessment of the real tumor burden, especially in the era of the sub-stratification between M0 and M1 settings, as well as castration-sensitive and castration-resistant phases. Assuming prostate cancer natural history like an iceberg of which we often see only the tip, in recent years several scientific experiences have been reported to introduce an imaging method able to show a larger proportion of this iceberg (3).

The growing availability of ${ }^{18} \mathrm{~F}$-Fluciclovine and ${ }^{68} \mathrm{Ga}$ PSMA is providing new precious tools for the urooncologic community to assess the real extent of the disease and thus orient the best therapeutic strategy in patients with low post-prostatectomy PSA blood levels $(\leq 1 \mathrm{ng} / \mathrm{mL})$ (4).

Compared to conventional imaging and to other tracers like ${ }^{11} \mathrm{C}$-Choline, more suitable for higher PSA levels (5), both tracers have collected superior sensitivity and detection rates for lower PSA values. Given the relatively recent introduction in the medical field, to date, there is only one study that reports a direct prospective comparison between them.

The study recently published by Calais et al. on The Lancet Oncology (6) concerns a prospective monoinstitutional series of 50 consecutive paired PET-CT scans performed with ${ }^{18} \mathrm{~F}$-Fluciclovine and ${ }^{68} \mathrm{Ga}$-PSMA in the setting of biochemical recurrence after surgery. Primary endpoint was the detection rate, with the aim to assess an expected difference of at least $22 \%$ in favor of ${ }^{68} \mathrm{Ga}$-PSMA, while secondary endpoints were detection rates stratified by PSA values (ranged from 0.2 to $2.0 \mathrm{ng} / \mathrm{mL}$ ), positive predictive value and sensitivity of both tracers, and, finally, inter-observer variability assessment.

The Authors reported a significantly $(\mathrm{P}=0.0026$; $\mathrm{OR}=4.8)$ higher detection rate of ${ }^{68} \mathrm{Ga}$-PSMA [28 patients out of 50 (56\%; 95\% CI: $41-70 \%)$ ] compared to ${ }^{18} \mathrm{~F}$-Fluciclovine [13 cases out of 50 (26\%; 95\% CI: $15-40 \%)$ ], thus reaching the primary endpoint. Therefore, in a subgroup analysis by regional level of recurrence, ${ }^{68} \mathrm{Ga}$-PSMA showed superior rates of pelvic lymph nodes metastases detection [4 cases detected by ${ }^{18} \mathrm{~F}$-Fluciclovine vs. 15 diagnosed by ${ }^{68} \mathrm{Ga}$-PSMA (P=0.0034; OR =12)] and for all extrapelvic secondary lesions $\left[0\right.$ cases with ${ }^{18} \mathrm{~F}$-Fluciclovine $v s .8$ with 
${ }^{68} \mathrm{Ga}$-PSMA ( $\mathrm{P}=0.0078$; OR = not estimable)], while no difference was observed for prostate fossa $(\mathrm{P}=0.73)$ and for extrapelvic lesions divided by site (bone, extra pelvic nodes or other organs), despite the M1-population data might be affected by the small number of patients.

A further subgroup analysis was performed basing on PSA levels categories $(0.2-0.5,0.51-1$ and $1.01-2 \mathrm{ng} / \mathrm{mL})$, collecting no significant differences in terms of detection rate, most likely due to the small sample size. Lastly, the inter-observer agreement was consistently superior with ${ }^{68} \mathrm{Ga}$-PSMA compared to ${ }^{18} \mathrm{~F}$-Fluciclovine $(\mathrm{P}=0.0020)$ for both PSA levels and regional sites of recurrence, with the exception of prostate bed.

The series presented by Calais et al. supports the use of ${ }^{68} \mathrm{Ga}-\mathrm{PSMA}$ as the tracer of choice for the early biochemical recurrence setting, since it showed superior reliability for the detection of pelvic or extrapelvic metastatic lesions, with a lower inter-observer variation. The Authors address these results to some main factors; first of all, the higher expression of PSMA in the hormone-sensitive scenario has led to doubly higher SUVmax values compared to ${ }^{18} \mathrm{~F}$-Fluciclovine; secondly, unlike the protein LAT-1 which is the main carrier of Fluciclovine into tumor cells, PSMA is poorly expressed by non-target areas, thus resulting in a major contrast with the normal tissue background, which also contributes to increasing the readers' agreement.

Of note, ${ }^{68}$ Ga-PSMA global widespread may have produced higher levels of confidence in reading compared to ${ }^{18}$ F-Fluciclovine, which is mostly used in US; furthermore, the protocol for ${ }^{18} \mathrm{~F}$-Fluciclovine uptake time was shorter than recommended by the American College of Nuclear Medicine Guidelines (2 versus 3-5 min), thus representing two potential biases of the study.

A significant difference between the two tracers failed to be found in a subgroup analysis by PSA levels probably due to the small number of patients enrolled. Nonetheless, PSMA is reported to outperform other tracers in the presence of PSA $<0.5 \mathrm{ng} / \mathrm{mL}$ with detection rates up to $58 \%$ in some series (7).

The subgroup analysis by regional site revealed a profound superiority of ${ }^{68} \mathrm{Ga}$-PSMA compared to ${ }^{18} \mathrm{~F}$-Fluciclovine in detecting both pelvic or extra-pelvic metastases: despite the study was not designed for the evaluation of the impact of PET findings on patient management, this evidence confirms the crucial need for an accurate method for the detection of any potential extra-prostatic disease in order to plan the best tailored therapeutic approach.
Keeping in mind all the above-mentioned limitations, the findings of the study by Calais et al. are of main importance in light of the growing evidence supporting the role of ${ }^{68} \mathrm{Ga}-\mathrm{PSMA}-\mathrm{PET}$ in the biochemical recurrence setting, as also recommended by the German $S 3$ and the European Association of Urology guidelines $(8,9)$.

Several Authors also report the use of ${ }^{68} \mathrm{Ga}$-PSMA-PET for the definition of potential recurrence patterns, particularly for pelvic lymph nodes, proposing the use of this tracer as a guide for treatment volumes delineation (10), hypothesizing a future change in the current standard contouring guidelines for pelvic lymph nodes radiotherapy $(11,12)$.

Notably, the role of pelvic radiotherapy in the postoperative patient is still controversial, and its impact on clinical outcomes is currently unclear (13).

New data are awaited from two randomized clinical trials investigating the role of both ${ }^{18} \mathrm{~F}$-Fluciclovine and ${ }^{68} \mathrm{Ga}$-PSMA in salvage radiotherapy (NCT03582774 and NCT03762759). However, it is reasonable to presume that a negative ${ }^{68} \mathrm{Ga}$-PSMA-PET may be the ideal trigger for selecting the real biochemical recurrent patient who benefits from prostate bed salvage radiotherapy, also for cases with PSA values $<0.5 \mathrm{ng} / \mathrm{mL}$ (14).

This is also confirmed by Boreta et al. (15) who observed a change of planning for salvage radiotherapy in $41.3 \%$ of cases due to PET findings, suggesting that the main role of ${ }^{68} \mathrm{Ga}$-PSMA can be attributed to the exclusion of metastatic disease.

Conversely, also De Bari et al. (16) highlight the use of ${ }^{68}$ Ga-PSMA-PET as a crucial decision-making tool to detect oligo-metastatic patients potentially candidate for a metastasis-directed treatment (MDT), despite to date, long term follow-up data of PSMA-PET-guided MDT are still awaited.

The use of stereotactic body radiotherapy (SBRT) in the setting of oligo-metastatic disease is currently an attractive option, particularly in the castration-sensitive scenario, where clinicians may offer a therapeutic alternative to the premature start of androgen deprivation therapy. Nevertheless, the role of SBRT in the oligo-metastatic prostate cancer is still under investigation in terms of impact on clinical outcomes, although preliminary encouraging results have been recently published, also including the castration-resistant population (17-21).

In fact, despite these new tracers may allow the early detection of small metastases amenable for local treatments, there might be an amount of undetectable microscopic disease, potentially underestimated also by ${ }^{68} \mathrm{Ga}$-PSMA- 
PET (22).

Keeping in mind that PSMA-sensitivity is PSArelated, still, it has produced a remarkable reduction of the proportion of the so-called "indemonstrable minimal residual disease" (23).

To keep pursuing this goal, future strategies must be oriented towards two main objectives: first, the improvement of imaging quality especially in terms of spatial resolution, as it may be enhanced by the future widespread implementation of MRI-based PET scans, for which first experiences describe higher detection rates compared to the PET-CT imaging, respectively of $44 \%$ (for PSA $<0.2 \mathrm{ng} / \mathrm{mL}$ ) and $72.7 \%$ (for PSA ranging between 0.2 and $0.5 \mathrm{ng} / \mathrm{mL}$ ) (24).

Secondly, some studies report preliminary experiences aimed to improve the sensitivity of PSMA-PET basing on the use of other ligands than ${ }^{68}$ Gallium like ${ }^{18}$ Fluorine, which seems to increase the detection rates for smaller targets even with PSA values between 0.2 and $0.49 \mathrm{ng} /$ $\mathrm{mL}$. Furthermore, given the lower urinary excretion of ${ }^{18}$ Fluorine, also local recurrence detection rates seem to be improved compared to ${ }^{68}$ Gallium-based PET scans (25).

The study of Calais et al. endorses the use of ${ }^{68} \mathrm{Ga}$ PSMA as the tracer of first choice in biochemical recurrent prostate cancer, with a consequent potential favorable impact on the therapeutic algorithm in this scenario. The superior detection rates for lower PSA values allow the early identification of potential metastatic sites, and on this basis, provide a trustworthy tool for the assessment of the disease extent, in which clinicians may rely to propose the best tailored therapeutic approach.

Still, a proportion of micro-metastatic disease remains occult, since smaller lesions detection remains an unsolved issue. However, compared to the recent past conventional imaging, new tracers may lead to a lowering of the tip of the prostate cancer iceberg, by showing with higher accuracy the real tumor burden, thus tracing the pathway to the melting of the iceberg as a viable goal for the future.

\section{Acknowledgments}

None.

\section{Footnote}

Conflicts of Interest: The authors have no conflicts of interest to declare.

Ethical Statement: The authors are accountable for all aspects of the work in ensuring that questions related to the accuracy or integrity of any part of the work are appropriately investigated and resolved.

\section{References}

1. Fossati N, Gandaglia G, Briganti A, et al. The emerging role of PET-CT scan after radical prostatectomy: still a long way to go. Lancet Oncol 2019;20:1193-5.

2. National Comprehensive Cancer Network. Guidelines for treatment of cancer by site: prostate cancer. Version 4.2019. Available online: https://www.nccn.org/ professionals/physician_gls/pdf/prostate.pdf

3. De Visschere PJL, Standaert C, Fütterer JJ, et al. A Systematic Review on the Role of Imaging in Early Recurrent Prostate Cancer. Eur Urol Oncol 2019;2:47-76.

4. Rayn KN, Elnabawi YA, Sheth N. Clinical implications of PET/CT in prostate cancer management. Transl Androl Urol 2018;7:844-54.

5. Alongi F, Fersino S, Giaj Levra N, et al. Impact of 18F-Choline PET/CT in the Decision-Making Strategy of Treatment Volumes in Definitive Prostate Cancer Volumetric Modulated Radiation Therapy. Clin Nucl Med 2015;40:e496-500.

6. Calais J, Ceci F, Eiber M, et al. (18)F-fluciclovine PETCT and (68)Ga-PSMA-11 PET-CT in patients with early biochemical recurrence after prostatectomy: a prospective, single-centre, single-arm, comparative imaging trial. Lancet Oncol 2019;20:1286-94.

7. van Leeuwen PJ, Stricker P, Hruby G, et al. (68) GaPSMA has a high detection rate of prostate cancer recurrence outside the prostatic fossa in patients being considered for salvage radiation treatment. BJU Int 2016;117:732-9.

8. EAU Guidelines. Edn. presented at the EAU Annual Congress Barcelona 2019. ISBN 978-94-92671-04-2.

9. Interdisziplinäre Leitlinie der Qualität S3 zur Früherkennung, Diagnose und Therapie der verschiedenen Stadien des Prostatakarzinoms [Internet]. Available online: https://www.leitlinienprogramm-onkologie.de/leitlinien/ prostatakarzinom/ Accessed 9 May 2018.

10. Farolfi A, Ceci F, Castellucci P et al. (68)Ga-PSMA-11 $\mathrm{PET} / \mathrm{CT}$ in prostate cancer patients with biochemical recurrence after radical prostatectomy and PSA $<0.5 \mathrm{ng} /$ $\mathrm{ml}$. Efficacy and impact on treatment strategy. Eur J Nucl Med Mol Imaging 2019;46:11-9.

11. Fersino S, Tebano U, Mazzola R, et al. Moderate Hypofractionated Postprostatectomy Volumetric 
Modulated Arc Therapy With Daily Image Guidance (VMAT-IGRT): A Mono-institutional Report on Feasibility and Acute Toxicity. Clin Genitourin Cancer 2017;15:e667-73.

12. Zschaeck S, Lohaus F, Beck M, et al. PSMA-PET based radiotherapy: a review of initial experiences, survey on current practice and future perspectives. Radiat Oncol 2018;13:90.

13. De Bari B, Mazzola R, Aiello D, et al. ((68)Ga)-PSMAPET/CT for the detection of postoperative prostate cancer recurrence: Possible implications on treatment volumes for radiation therapy. Cancer Radiother 2019;23:194-200.

14. Emmett L, van Leeuwen PJ, Nandurkar R, et al. Treatment Outcomes from (68)Ga-PSMA PET/CTInformed Salvage Radiation Treatment in Men with Rising PSA After Radical Prostatectomy: Prognostic Value of a Negative PSMA PET. J Nucl Med 2017;58:1972-6.

15. Boreta L, Gadzinski AJ, Wu SY, et al. Location of Recurrence by Gallium-68 PSMA-11 PET Scan in Prostate Cancer Patients Eligible for Salvage Radiotherapy. Urology 2019;129:165-71.

16. De Bari B, Mazzola R, Aiello D, et al. Could 68-Ga PSMA PET/CT become a new tool in the decision-making strategy of prostate cancer patients with biochemical recurrence of PSA after radical prostatectomy? A preliminary, monocentric series. Radiol Med 2018;123:719-25.

17. Siva S, Bressel M, Murphy DG, et al. Stereotactic Abative Body Radiotherapy (SABR) for Oligometastatic Prostate Cancer: A Prospective Clinical Trial. Eur Urol 2018;74:455-62.

18. Triggiani L, Alongi F, Buglione M, et al. Efficacy of stereotactic body radiotherapy in oligorecurrent and in

Cite this article as: Mazzola R, Cuccia F, Figlia V, Giaj-Levra N, Nicosia L, Ricchetti F, Rigo M, Pasinetti N, Salgarello M, Alongi F. New metabolic tracers for detectable PSA levels in the post-prostatectomy setting: is the era of melting glaciers upcoming? Transl Androl Urol 2019;8(Suppl 5):S538-S541. doi: 10.21037/tau.2019.12.34 oligoprogressive prostate cancer: new evidence from a multicentric study. Br J Cancer 2017;116:1520-5.

19. Ost P, Reynders D, Decaestecker K, et al. Surveillance or Metastasis-Directed Therapy for Oligometastatic Prostate Cancer Recurrence: A Prospective, Randomized, Multicenter Phase II Trial. J Clin Oncol 2018;36:446-53.

20. Triggiani L, Mazzola R, Magrini SM, et al. Metastasisdirected stereotactic radiotherapy for oligoprogressive castration-resistant prostate cancer: a multicenter study. World J Urol 2019;37:2631-7.

21. Radwan N, Phillips R, Ross A, et al. A phase II randomized trial of Observation versus stereotactic ablative RadiatIon for OLigometastatic prostate CancEr (ORIOLE). BMC Cancer 2017;17:453.

22. Nicosia L, Franzese C, Mazzola R, et al. Recurrence pattern of stereotactic body radiotherapy in oligometastatic prostate cancer: a multi-institutional analysis. Strahlenther Onkol 2019. [Epub ahead of print].

23. Murray NP. Minimal residual disease in prostate cancer patients after primary treatment: theoretical considerations, evidence and possible use in clinical management. Biol Res 2018;51:32.

24. Verburg FA, Pfister D, Heidenreich A, et al. Extent of disease in recurrent prostate cancer determined by [(68) Ga]PSMA-HBED-CC PET/CT in relation to PSA levels, PSA doubling time and Gleason score. Eur J Nucl Med Mol Imaging 2016;43:397-403.

25. Luiting HB, van Leeuwen PJ, Busstra MB, et al. Use of gallium-68 prostate-specific membrane antigen positronemission tomography for detecting lymph node metastases in primary and recurrent prostate cancer and location of recurrence after radical prostatectomy: an overview of the current literature. BJU Int 2019. [Epub ahead of print]. 